\title{
Effect of Orally Administered Microencapsulated FA-Producing L. fermentum on Markers of Metabolic Syndrome: An In vivo Analysis
}

\section{Jasmine Bhathena", Catherine Tomaro-Duchesneau\#, Christopher Martoni, Meenakshi Malhotra, Arun Kulamarva, Aleksandra Malgorzata} Urbanska, Arghya Paul and Satya Prakash*

Biomedical Technology and Cell Therapy Research Laboratory, Departments of Biomedical Engineering, Physiology, and Artificial Cells and Organs Research Center, McGill University, Canada

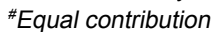

\begin{abstract}
Ferulic Acid (FA) is a natural phenolic acid produced by a number of lactic acid bacteria. FA has a number of beneficial properties, including: antioxidant activity, anti-tumorigenic properties and cholesterol-lowering capabilities. Our group has previously screened lactobacilli for FA production, and selected L. fermentum ATCC 11976 (L.f. 11976 ) as one of the best producers. Alginate-polylysine-alginate (APA) microencapsulation has proven successful for the oral delivery of this strain to the colon, where production of FA is greatest. The aim of this study was to investigate the role of APA microencapsulated L.f. 11976 to modulate markers of metabolic syndrome. The antioxidant activity, as a potential mechanism of action to treat/prevent metabolic syndrome of free and microencapsulated L.f. 11976 was quantified. A high-fat fed BioF $_{1} B$ Golden Syrian hamster model was used to investigate the effects of orally administered microencapsulated L.f. 11976 on markers of metabolic syndrome. Results demonstrate that the microencapsulated L.f. 11976 formulation greatly reduced the adiposity index $(p=0.0014)$, serum insulin $(p=$ $0.0042)$, insulin resistance $(p=0.0096)$, glycosylated albumin $(p=0.00013)$, serum leptin $(p=0.048)$, serum uric acid $(p=0.025)$ serum total cholesterol $(p=0.024)$, serum esterified cholesterol $(p=0.0328)$ and free non-esterified fatty acid $(p=0.029)$ levels in the treated animals. This research indicates that the probiotic L.f. 11976 microencapsulated formulation may significantly delay the onset of insulin resistance, hyperglycemia, hyperinsulinemia, dyslipidemia and obesity, indicating a lower risk of diabetes and cardiovascular disease. We propose and discuss the potential mechanism(s) of action by which FA is acting. With these in mind, further in vivo studies are required to validate the therapeutic effects of the formulation and to investigate the mechanism(s) of action by which the probiotic formulation is acting.
\end{abstract}

Keywords: Ferulic acid; Microencapsulation; Metabolic syndrome; Lactobacillus; Type-2-diabetes mellitus; Obesity

Abbreviations: FA: Ferulic Acid; APA : Alginate-Polylysine-Alginate; L.f. 11976 : Lactobacillus fermentum ATCC 11976; T2DM : Type 2 Diabetes Mellitus; GIT: Gastrointestinal Tract; FAE: Feruloyl Esterase; EFA: Ethyl Ferulate; MRS: De Man-Rogosa-Sharpe; TC: Total Cholesterol; HbA1c: Glycosylated Hemoglobin; CRP: C-Reactive Protein; FC: Free Cholesterol; NEFA: Non-Esterified Fatty Acids; GA: Glycosylated Albumin; HOMA-IR: Homeostasis Model Assessment (for) Insulin Resistance; EC: Esterified Cholesterol; LDL: Low Density Lipoprotein Cholesterol; TG: Triglycerides

\section{Introduction}

Ferulic acid [trans-4-hydroxy-3-methoxycinnamic acid] (FA) is a naturally found phenolic acid that is abundantly bound to most of the foods consumed by humans (bran, whole grains, fruits, vegetables, tea, coffee). This molecule is a potent antioxidant able to neutralize free radicals, such as Reactive Oxygen Species (ROS) [1] which have been implicated in DNA damage [2], cancer[3], accelerated cell aging [4], obesity [5] and type 2 diabetes mellitus (T2DM) [5,6]. A number of gastrointestinal tract (GIT) bacterial strains have been shown to produce feruloyl esterase (FAE) [7-12], an enzyme that hydrolyses and releases free FA from its bound state. Orally ingested FA-producing lactic acid bacteria should demonstrate an increased bioavailability of FA due to increased FAE activity in the intestine. Live microorganisms have been ingested and fermented in foods for centuries, with Metchnikoff establishing the concept of probiotics in the early 1900s [13]. Unfortunately, probiotic survival incorporated in foods is usually low due to the harsh environment (low pH, digestive enzymes, bile) of the GIT. Microencap- sulation in polymer membranes, such as alginate-polylysine-alginate (APA) has been shown to successfully protect live bacterial cells for oral delivery $[8,11,14]$. Specifically, the microencapsulation of FAE bacteria has demonstrated a higher viable cell count delivery through the GIT, allowing for a higher FA bioavailability $[8,9,11]$.Previous research by our group has demonstrated that microencapsulated Lactobacillus fermentum ATCC 11976 (L.f. 11976) can be used as a probiotic formulation in the context of cholesterol management in a Bio F1B hypercholesterolemic Golden Syrian hamster model [9].

The increased bioavailability of FA in the GIT may also induce additional metabolic changes which should prove beneficial for the treatment of metabolic syndrome, specifically T2DM, obesity and cardiovascular diseases. T2DM and obesity are two major public health concerns in industrialized countries. The prevalence of diabetes in the United States in 2005 was estimated at 16.2 million individuals and is projected

*Corresponding author: Satya Prakash, Biomedical Technology and Cell Therapy Research Laboratory, Department of Biomedical Engineering and Artificia Cells and Organs Research Centre, Faculty of Medicine, McGill University, 3775 University Street, Montreal, Québec, H3A 2B4, Canada, Tel: 1514-398-3676; Fax: 1514-398-7461; E-mail: satya.prakash@mcgill.ca

Received April 25, 2012; Accepted May 20, 2012; Published May 25, 2012

Citation: Bhathena J, Tomaro-Duchesneau C, Martoni C, Malhotra M, Kulamarva A, et al. (2012) Effect of Orally Administered Microencapsulated FA-Producing $L$. fermentum on Markers of Metabolic Syndrome: An In vivo Analysis. J Diabetes Metab S6:006. doi:10.4172/2155-6156.S6-006

Copyright: (c) 2012 Bhathena J, et al. This is an open-access article distributed under the terms of the Creative Commons Attribution License, which permits unrestricted use, distribution, and reproduction in any medium, provided the original author and source are credited. 
Citation: Bhathena J, Tomaro-Duchesneau C, Martoni C, Malhotra M, Kulamarva A, et al. (2012) Effect of Orally Administered Microencapsulated FA-Producing L. fermentum on Markers of Metabolic Syndrome: An In vivo Analysis. J Diabetes Metab S6:006. doi:10.4172/2155-6156.S6-006

to rise to 48.3 million individuals by 2050 [15]. Obesity, also related to $\mathrm{T} 2 \mathrm{DM}$, is an epidemic, not only because of its increasing prevalence but, also because of its frequent association with major atherosclerotic and cardiovascular risk factors [16]. As a potential natural therapeutic, FA has been shown to regulate blood glucose levels through a variety of mechanisms including the modulation of insulin secretion, the promotion of pancreatic beta-cell survival and the reduction of inflammatory markers linked to antioxidant activity [17]. With these properties in mind, FA could provide a way to modulate hyperglycemia, hyperinsulinema, adiposity and other markers involved in a number of metabolic diseases, including T2DM and obesity.

The presented research attempts to demonstrate the antioxidant properties of a commercially available FA, to subsequently investigate the antioxidant potential of L.f. 11976, associated with its growth in ethyl ferulate (EFA), a natural substrate of FAE. Furthermore, we investigate, in vivo, the use of the FA-producing L.f. 11976 microencapsulated formulation to modulate markers involved in the pathogenesis of metabolic disorders such as T2DM and obesity using a diet-induced hypercholesterolemic hamster model.

\section{Materials and Methods}

\section{Bacterial growth media and chemicals}

EFA (ethyl 4-hydroxy-3-methoxycinnamate), FA, poly-l-lysine, calcium chloride and low viscosity sodium alginate were purchased from Sigma-Aldrich (Oakville, ON, Canada). De Man-Rogosa-Sharpe (MRS) broth was obtained from Fisher Scientific (Ottawa, ON, Canada). Water was purified with an EASYpure Reverse Osmosis System and a NANO pure Diamond Life Science (UV/UF) ultrapure water system from Barnstead/Thermoline (Dubuque, IA, U.S.A.). All other chemicals were of analytical or High-Performance Liquid Chromatrography (HPLC) grade and purchased from commercial sources.

\section{Bacterial strain and culture conditions}

L.f. 11976 was purchased from Cedarlane Laboratories (Burlington, $\mathrm{ON}$, Canada). The bacterial strain was stored at $-80^{\circ} \mathrm{C}$ in MRS containing $20 \%(\mathrm{v} / \mathrm{v})$ glycerol. An MRS-agar plate was streaked for isolation from the frozen stock and incubated at $37^{\circ} \mathrm{C}$ with $5 \% \mathrm{CO}_{2}$ for $24 \mathrm{~h}$ to ensure purity. One colony from the MRS-agar plate was inoculated into $5 \mathrm{~mL}$ of MRS broth and incubated at $37^{\circ} \mathrm{C}$ for $24 \mathrm{~h}$. A $1 \%(\mathrm{v} / \mathrm{v}$ ) inoculum was then used for subculturing and incubated at $37^{\circ} \mathrm{C}$ for $24 \mathrm{~h}$ immediately before use. Viability of microcapsules was estimated by exposure to $0.1 \mathrm{M}$ sodium citrate until complete disruption of the microcapsules was observed. Ten-fold serial dilutions in physiological saline followed by plating on MRS-agar plates were then performed to determine the colony forming units.

\section{APA microencapsulation of L.f. 11976}

The microencapsulation of L.f. 11976, using APA polymer, was performed according to the standard protocol, described in our previous studies $[8,11]$. The microencapsulation procedure was performed using an Inotech encapsulator (Inotech Biosystems International, MD, U.S.A.) and optimal flow rate, vibration frequency and voltage. The semi-permeable microcapsules obtained have a molecular weight cutoff of $60-70 \mathrm{kDa}$, as previously demonstrated [8]. Microcapsules termed "empty," which do not contain bacteria were also prepared as controls. Microencapsulated lactobacilli were stored at $4^{\circ} \mathrm{C}$, in minimal media consisting of $10 \%$ MRS until further use. Previous work demonstrated no significant loss of cell viability or changes in microcapsule stability using this storage method [7].

\section{Total antioxidant capacity of $L . f .11976$}

The total antioxidant production of L.f. 11976 was measured using a QuantiChrom ${ }^{\mathrm{TM}}$ Antioxidant Assay Kit (BioAssay systems, CA, U.S.A.), a spectrophotometric assay based on the reduction of $\mathrm{Cu}^{+}$to $\mathrm{Cu}^{+}$measured using a UV spectrophotometer Victor ${ }^{3} \mathrm{~V} 1420$ Multi label Counter (Perkin Elmer, MA, U.S.A.). The protocol provided with the assay kit was followed. A standard curve was generated for Trolox, a standard provided with the kit, at concentrations of $0,300,600$ and $1000 \mu \mathrm{M}$ plotted against absorbance at $570 \mathrm{~nm}\left(\mathrm{R}^{2}=0.9970\right)$. Antioxidant activity of commercially available FA at concentrations of $0.3,0.6,0.9,1.2$ and $1.5 \mathrm{mM}$ was plotted against Trolox concentrations at an absorbance of $570 \mathrm{~nm}$. Unencapsulated L.f. 11976 was subcultured from MRS broth at $1 \%(\mathrm{v} / \mathrm{v})$ and microencapsulated L.f. 11976 at a concentration of $0.05 \mathrm{~g} /$ $\mathrm{mL}$ was added to MRS-EFA broth at an EFA concentration of $1.5 \mathrm{mM}$. Uninoculated MRS-EFA broth was used as a negative control. At each time point, the viability of the microcapsules and free cells was determined using ten-fold serial dilutions in physiological saline followed by plating on MRS-agar plates. Bacterial supernatant was obtained from each sample by centrifuging at $4000 \mathrm{rpm}$ at $4^{\circ} \mathrm{C}$ for 15 minutes. The supernatant was stored at $4^{\circ} \mathrm{C}$ until the assay was performed. All samples and controls were treated in triplicate and incubated at $37^{\circ} \mathrm{C}$ during the course of the experiment.

\section{Animals}

Male Bio F B Golden Syrian hamsters, 8 weeks old (BioBreeders, MA, U.S.A.) with an average body weight of $90 \mathrm{~g}$ were used in this study. The animals were housed three per cage in a climate controlled space with inversed, alternating light and dark cycles (12:12-hr lightdark cycle; lights on at 19:00). All the principles of laboratory animal care were followed and all of the experimental protocols complied with the Animal Care Committee of McGill University (Montréal, Canada) and the Canadian Council on Animal Care guidelines.

\section{Animal diet and experimental protocol}

Upon their arrival, animals were allowed free access to a commercial rodent ration (LabDiet ${ }^{\circledR}$ Rodent Laboratory Chow 5001, Purina Laboratories, St. Louis, MO, U.S.A.) and water for 2 weeks during their acclimatization period. Baseline values of serum Total Cholesterol (TC) were determined, as described below, at the end of the acclimatization from hamsters fasted over a 14-hour period. These basal serum TC values were used to randomize the hamsters into the two treatment groups.

Following randomization, the hamsters of both groups were fed a semi-purified hyperlipidemic hypercholesterolemic diet ad libitum (Modified LabDiet ${ }^{\circledR}$ Laboratory Rodent Diet 5001 with $10.0 \%$ Wheat Bran, 0.05\% Cholesterol, 6.0\% Saturated Fat and 10.0\% Total Fat, Purina Laboratories, St. Louis, MO, U.S.A.). To investigate the therapeutic efficacy of the formulation, animals in the treatment group $(n=12)$ received the microcapsule formulation containing $10^{11} \mathrm{cfu}$ of L.f. 11976 twice daily, a dose determined from previous work by our group [9]. The control group $(n=12)$ received empty microcapsules as treatment vehicle-control. The treatment was administered during 10 weeks, with the hamsters orally fed the formulation using an $18 \mathrm{G} / 50 \mathrm{~mm}$ with $2.25 \mathrm{~mm}$ ball diameter stainless steel gavage needle. During the course of the treatment, food consumption and body weight were measured biweekly. Blood samples were collected once every 14 days from 14hour food-deprived hamsters via the saphenous vein. At the end of the experimental period, the hamsters were euthanized by carbon dioxide asphyxiation and blood was withdrawn by cardiac puncture. 


\section{Biological samples}

Blood was collected either in Microtainer ${ }^{\circledR}$ serum separator tubes or in Vacutainer ${ }^{\oplus}$ plasma tubes with spray-coated lithium heparin from Fisher Scientific (Ottawa, ON, Canada). For serum, the blood was allowed to clot at $23^{\circ} \mathrm{C}$ for 30 minutes and subsequently placed on ice until centrifugation. Serum was separated by low-speed centrifugation at $2000 \mathrm{xg}$ for $20 \mathrm{~min}$ at $4^{\circ} \mathrm{C}$ temperature and stored at $-80^{\circ} \mathrm{C}$ until used. To calculate the adiposity index, the weights of the epididymal, visceral and retroperitoneal fat were taken following their careful dissection. The adiposity index was calculated using the formula [Visceral fat/ (body weight-visceral fat) ${ }^{*} 100$, and expressed as adiposity percentage [18].

\section{Clinical chemistry analysis}

Serum lipids (TC, triglycerides), glucose, uric acid, glycosylated hemoglobin (HbAlc) and C-Reactive Protein (CRP) were assayed by conventional enzymatic methods on a Hitachi 911 automated clinical chemistry autoanalyzer (Roche Diagnostics, U.S.A.) using reagent kits supplied by Roche Diagnostics (Laval, QC, Canada). Serum Free Cholesterol (FC) and Non-Esterified Fatty Acids (NEFA) were similarly estimated using reagent kits from Wako Chemicals Inc. (Richmond, VA, U.S.A). Serum insulin and leptin were measured by an Enzyme Immunoassay kit from SPI-BIO (Massy, France). Glycosylated albumin (GA) was estimated using a Glycaben ELISA kit from Exocell Inc. (Philadelphia, PA, U.S.A.).Fasting serum insulin and glucose were used to calculate insulin resistance from the homeostasis model assessment for insulin resistance (HOMA-IR) [(fasting glucose * fasting insulin)/22.5] [19]. Serum cholesteryl ester (EC) concentrations were calculated as the difference between the TC and the FC concentrations.

\section{Statistical analysis}

Experimental results are expressed as mean \pm SEM. Statistical analysis was carried out using SPSS Version 17.0 (Statistical Product and Service Solutions, IBM Corporation, New York, NY, USA). Linear regression was performed to correlate FA and Trolox concentrations. Statistical comparisons were carried out using the Independent Samples $t$-test to compare the means of the treatment and control groups. Statistical significance was set at $\mathrm{p}<0.05$ and $\mathrm{p}$-values less than 0.01 were considered highly significant.

\section{Results}

\section{Microencapsulation of L.f. 11976}

L.f. 11976 microencapsulation was optimized-by controlling the flow rate, stirring time, stirring speed, coating time, vibration frequency, and voltage to obtain spherical and monodispersed microcapsules. The obtained L.f. 11976 microcapsules were observed under light microscopy at magnifications of $100 \mathrm{X}$ and $200 \mathrm{X}$. The APA microcapsules were monodispersed with an approximate size of $602 \pm 30 \mu \mathrm{m}$ in diameter. Figure $1 \mathrm{~A}$ demonstrates the spherical shape of the microcapsule and Figure 1B allows for the visualization of the outer coat of the APA microcapsule with the bacterial cells evenly distributed within the polymeric membrane.

Total antioxidant capacity of free and microencapsulated $L . f$. 11976

To investigate the antioxidative potential of the FA produced by L.f. 11976 and to ensure that the microcapsule does not inhibit this activity, a QuantiChrom ${ }^{\mathrm{TM}}$ Antioxidant Assay Kit was used. Commercially purchased FA was shown to have significant antioxidant activity, with a

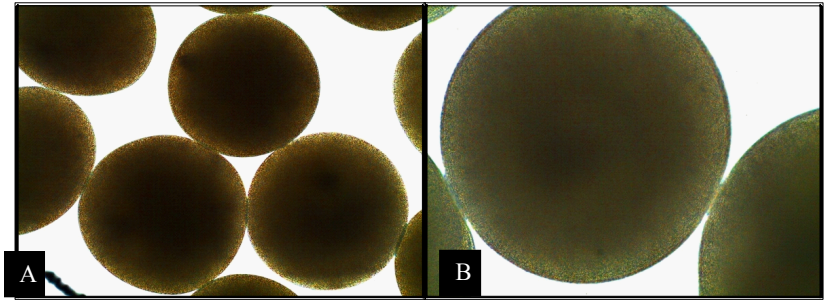

Figure 1: Morphology of APA microcapsules containing L.f. 11976 taken by light microscope (A) 100x and (B) 200x. The approximate diameter of the microcapsules was $602 \pm 30 \mu \mathrm{m}$ in diameter.

linear correlation demonstrated using Trolox as the provided standard $\left(\mathrm{R}^{2}=0.9881, p<0.0001\right)$ (Figure $\left.2 \mathrm{~A}\right)$. To quantify the bacterial antioxidant activity the probiotic strain was incubated in MRS-EFA at an initial EFA concentration of $1.5 \mathrm{mM}$. Antioxidant activity and bacterial viability were measured at various time points for both free (Figure $2 \mathrm{~B}$, 2C) and microencapsulated L.f. 11976 (Figure 2D, 2E). Following 48 hours of incubation, the antioxidant activity of L.f. 11976 was 713.81 $\pm 3.72 \mu \mathrm{M}$ Trolox equivalents for the free cells and $699.52 \pm 15.26 \mu \mathrm{M}$ Trolox equivalents for the microencapsulated cells. No significant difference in antioxidant production was detected between the free and microencapsulated cells $(p=0.450)$. Using the curve generated for FA the activity correlates to $1931.83 \pm 10.07 \mu \mathrm{M}$ for the free cells and $1893.15 \pm 41.30 \mu \mathrm{M}$ FA.

\section{Effect of microencapsulated L.f. 11976 on body weight and adiposity}

Dietary intake, food consumption and adiposity were monitored following the change to a hyperlipidemic hypercholesterolemic diet. The animals, of both groups, gained significant amounts of body weight over the course of the 10 week study (Figure 3A). Following the 10 weeks of treatment, there was no significant difference in body mass between the control $(157.20 \pm 2.25 \mathrm{~g})$ and the treatment $(151.42 \pm 3.17 \mathrm{~g})$ animals ( $p=0.178$ ).In addition, no significant difference in food consumption between the groups was observed (data not shown). As for the abdominal visceral fat weights, these were significantly lower in the animals treated with microencapsulated L.f. 11976.The adiposity index of the treated group of animals, at the end of the 10 weeks of treatment with microencapsulated L.f. $11976(6.22 \pm 0.25)$ proved to be significantly lower than that of the control animals $(7.94 \pm 0.35)(p=0.0014)$ (Figure 3B).

\section{Effect of microencapsulated $L . f .11976$ on serum glucose and insulin}

Fasting serum glucose was monitored throughout the treatment period and consumption of the hyperlipidemic hypercholesterolemic diet. Hamsters administered L.f. $11976(15.41 \pm 2.26 \mathrm{mmol} / \mathrm{L})$ showed no significant decrease in fasting serum glucose level following 10 weeks of treatment, when compared to the untreated control hamsters $(16.37 \pm$ $1.20 \mathrm{mmol} / \mathrm{L})(p=0.629)$ (Figure $4 \mathrm{~A})$. On the other hand, serum fasting insulin concentrations were significantly different between animals administered the microencapsulated bacterial formulation $(0.59 \pm 0.096$ $\mathrm{ng} / \mathrm{mL})$ and the controls $(1.40 \pm 0.217 \mathrm{ng} / \mathrm{mL})(p=0.0042)$ (Figure 4B). Moreover, intervention with the microcapsule formulation showed a significant reduction in insulin resistance (HOMA-IR) in treated animals $(66.51 \pm 10.78)$ as compared with the controls $(153.86 \pm 28.24)(p$ $=0.0096$ ) (Figure 4C). 
Citation: Bhathena J, Tomaro-Duchesneau C, Martoni C, Malhotra M, Kulamarva A, et al. (2012) Effect of Orally Administered Microencapsulated FA-Producing L. fermentum on Markers of Metabolic Syndrome: An In vivo Analysis. J Diabetes Metab S6:006. doi:10.4172/2155-6156.S6-006
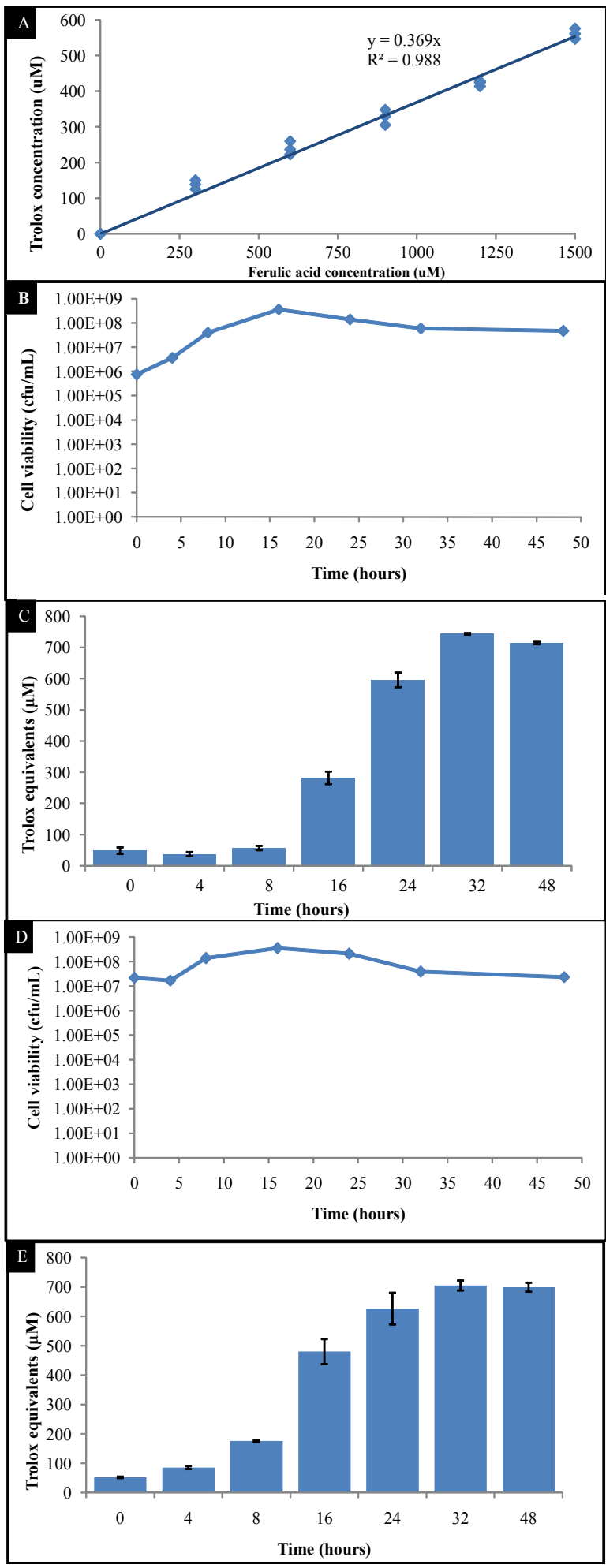

Figure 2: Antioxidant activity of ferulic acid produced by L.f. 11976. (A) Commercially purchased ferulic acid antioxidant activity correlated with Trolox concentration using a QuantiChrom ${ }^{\mathrm{TM}}$ Antioxidant Assay Kit $\left(\mathrm{R}^{2}=0.9881, p<\right.$ 0.0001). (B) Viability and (C) antioxidant production by free L.f. 11976 and (D) viability and (E) antioxidant production by APA microencapsulated L.f. 11976. Following 48 hours of incubation antioxidant activity of L.f. 11976 was $713.81 \pm$ $3.72 \mu \mathrm{M}$ Trolox equivalents for the free cells and 699.52 $\pm 15.26 \mu \mathrm{M}$ Trolox equivalents for the microencapsulated cells $(p=0.450)$

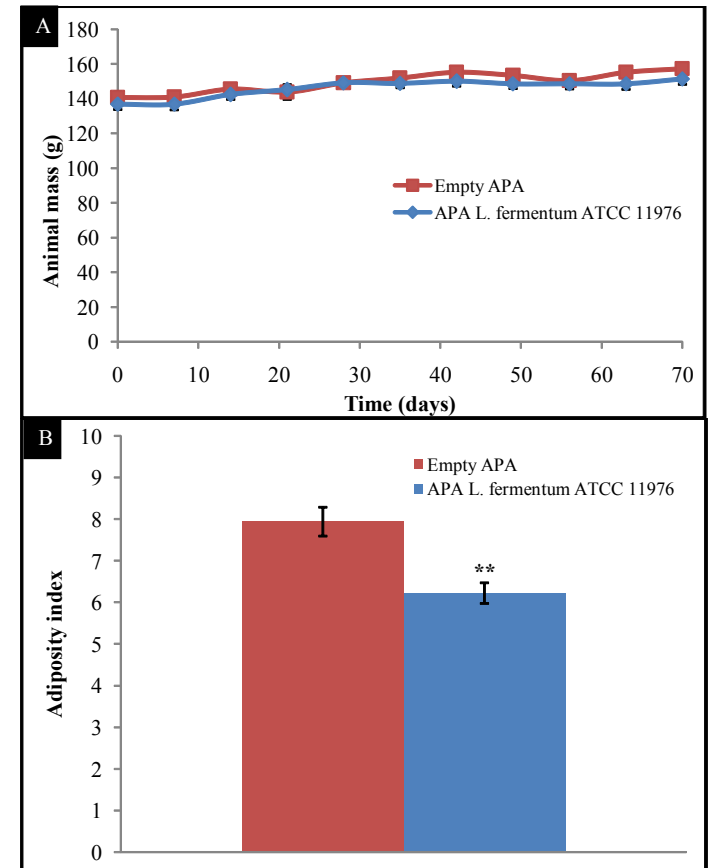

Figure 3: Effect of oral feeding of microencapsulated L.f. 11976 on the (A) animal mass over time and on the (B) adiposity index ( $\left.{ }^{* *} p<0.01\right)$ following 70 days of treatment in Golden Syrian hamsters. Data represents the mean \pm SEM $(n=12)$

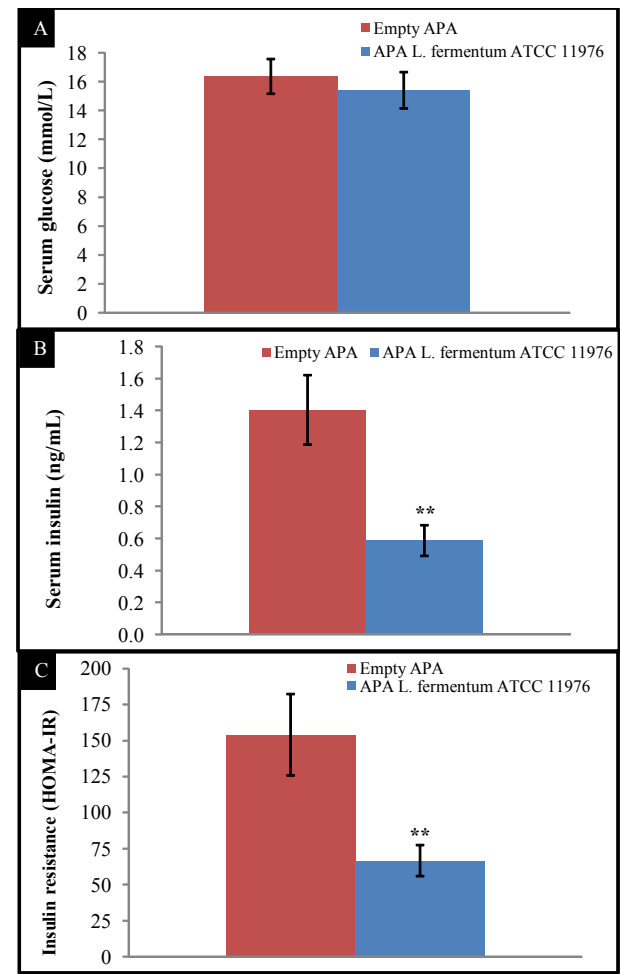

Figure 4: Treatment of diabetic obese Golden Syrian hamsters with microencapsulated L.f. 11976 resulted in a non-significant reduction in (A) serum glucose levels following 10 weeks of treatment, but showed a significant reduction in (B) serum insulin levels $\left({ }^{* *} p<0.01\right)$. (C) Insulin resistance was quantified using Homeostasis Model Assessment (HOMA-IR). The treated group showed HOMA-IR values significantly lower than untreated control animals $\left({ }^{* *} p<0.01\right)$. Data represents the mean \pm SEM $(n=12)$. 
Citation: Bhathena J, Tomaro-Duchesneau C, Martoni C, Malhotra M, Kulamarva A, et al. (2012) Effect of Orally Administered Microencapsulated FA-Producing L. fermentum on Markers of Metabolic Syndrome: An In vivo Analysis. J Diabetes Metab S6:006. doi:10.4172/2155-6156.S6-006

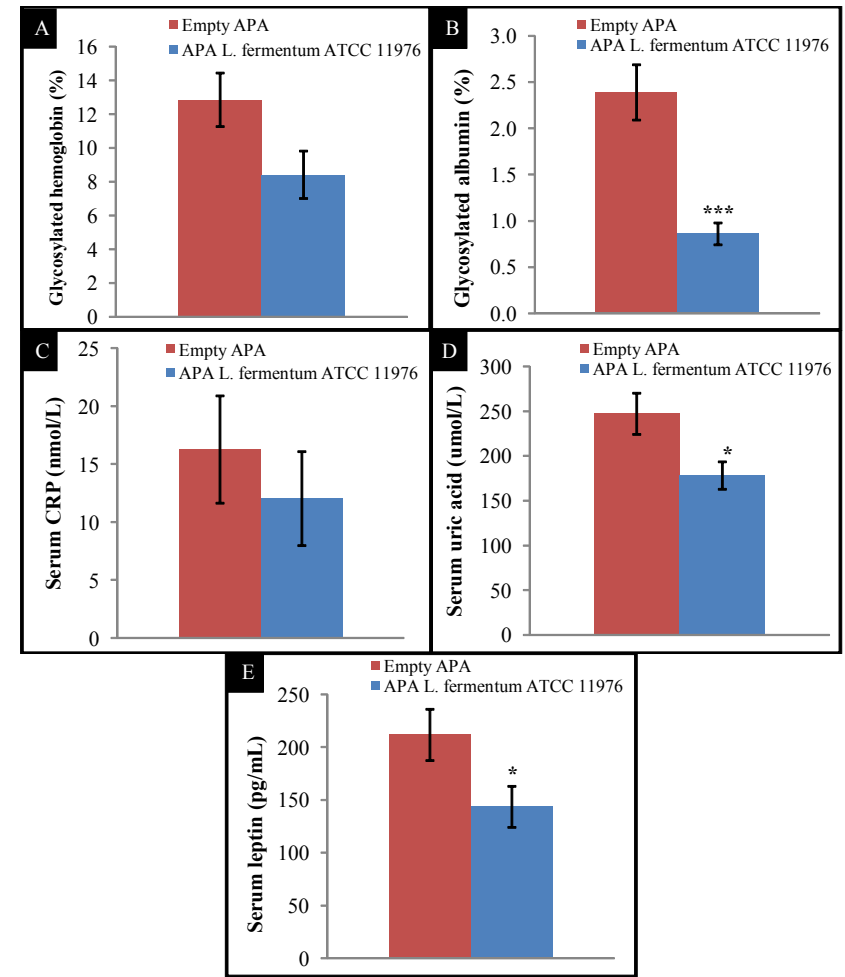

Figure 5: Changes in $(\mathrm{A})$ serum $\mathrm{HbA} 1 \mathrm{c},(\mathrm{B})$ serum $\mathrm{GA}\left({ }^{* * *} p<0.001\right),(\mathrm{C})$ serum $C R P,(D)$ serum uric acid $\left({ }^{*} p<0.05\right)$ and $(E)$ serum leptin $\left({ }^{*} p<0.05\right)$ levels in hamsters treated with microencapsulated L.f. 11976. Data represents the mean \pm SEM $(n=12)$.

\section{Effect of microencapsulated L.f. 11976 on glycemic control and inflammation}

Serum HbA1c levels were measured following 10 weeks of treatment with microencapsulated L.f. 11976. HbA1c was non-significantly reduced in the treated group $(8.41 \pm 1.40 \%)$ when compared to the control group $(12.85 \pm 1.59 \%)(p=0.058)$ (Figure $5 \mathrm{~A})$. On the other hand, a highly significant reduction in GA levels of the hamsters treated with microencapsulated L.f. $11976(0.86 \pm 0.12 \%)$ when compared with the control animals $(2.38 \pm 0.30 \%)$ was noted $(p=0.00013)$ (Figure $5 B)$.

Systemic inflammation, as measured by serum CRP levels, was not found to be significantly different between the treatment $(12.04 \pm 4.05$ $\mathrm{nmol} / \mathrm{L})$ and control $(16.27 \pm 4.62 \mathrm{nmol} / \mathrm{L})$ animals $(p=0.61)$ (Figure $5 \mathrm{C})$. On the other hand, the serum concentration levels of uric acid, following 10 weeks of treatment, were found to be significantly higher in the control group $(247.07 \pm 23.02 \mu \mathrm{mol} / \mathrm{L})$ when compared to the levels of the group administered microencapsulated L.f. 11976 (178.07 $\pm 15.32 \mu \mathrm{mol} / \mathrm{L})(p=0.025)$ (Figure $5 \mathrm{D})$. A statistically significant difference in the serum leptin levels was also noted between the treated $(143.54 \pm 19.46 \mathrm{pg} / \mathrm{mL})$ and the control $(211.75 \pm 24.33 \mathrm{pg} / \mathrm{mL})$ animals $(p=0.048)$ (Figure 5E).

\section{Effect of microencapsulated $L . f .11976$ on serum lipids}

Figure $6 \mathrm{~A}$ demonstrates that, following 10 weeks of treatment with microencapsulated L.f. 11976, there was a significant decrease $(p=0.024)$ in serum TC concentrations in the treated $(14.33 \pm 2.38$ $\mathrm{mmol} / \mathrm{L})$ as compared to the control $(23.18 \pm 2.52 \mathrm{mmol} / \mathrm{L})$ animals $(p=0.024)$. At the same time point, serum FC levels, did not show any difference between the treated $(1.71 \pm 0.31 \mathrm{mmol} / \mathrm{L})$ and the control $(1.55 \pm 0.33 \mathrm{mmol} / \mathrm{L})$ hamsters $(p=0.758)$. However, EC concentrations were significantly reduced in the treated animals $(12.91 \pm 2.51$ $\mathrm{mmol} / \mathrm{L})$ when compared to the control animals $(21.94 \pm 2.83 \mathrm{mmol} / \mathrm{L})$ $(p=0.0328)$.Figure 6B demonstrates that serum triglyceride (TG) concentrations were not significantly different between the treated $(6.28 \pm$ $0.97 \mathrm{mmol} / \mathrm{L})$ and the control $(8.36 \pm 0.51)$ groups $(p=0.0948)$. On the other hand, there was a significant reduction in serum NEFA concentrations in the treated animals $(2.65 \pm 0.12 \mathrm{mmol} / \mathrm{L})$ as compared to the control animals $(3.33 \pm 0.26 \mathrm{mmol} / \mathrm{L})(p=0.029)$.

\section{Discussion}

Probiotics are dietary supplements containing bacteria which, when administered in adequate amounts, confer a health benefit on the host $[14,20]$. Probiotics, as natural compounds, are generally considered safe, but can also be tested for set-out safety parameters [21]. A number of studies have investigated bacterial strains for a range of conditions, including infections, allergies and metabolic disorders such as ulcerative colitis and Crohn's disease [14]. Promising research focuses on the microbial secretion and production of beneficial biologically active enzymes and proteins, including: ornithine decarboxylase for the treatment of autoimmune diseases and accelerated cell apoptosis [22], bile salt hydrolase for hypercholesterolemia [23;24], and bile transport and tolerance proteins for the efficient delivery of probiotics [25]. In recent studies, the products of another microbial protein, cinnamoyl esterase, have shown significant levels of antioxidant activity $[10,26]$ and other effects, including stimulation of insulin secretion [17,27], prevention of oxidative stress [17], lipid peroxidation [28],cholesterol-lowering capabilities [9] and inhibition of diabetic nephropathy progression [29]. A product of hydrolysis activity of FAE,FA, is a well-characterised antioxidant [10].

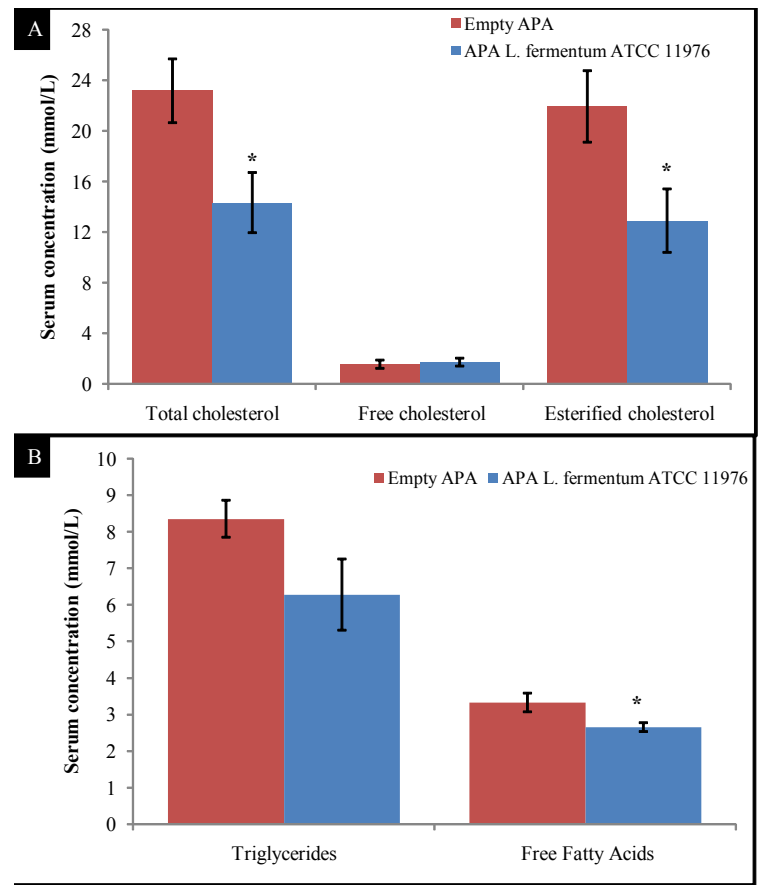

Figure 6: Clinical effects of microencapsulated L.f. 11976 on lipid metabolism in Golden Syrian hamsters; (A) serum total cholesterol ( $\left.{ }^{*} p<0.05\right)$, serum free cholesterol and esterified cholesterol $\left({ }^{*} p<0.05\right)$ and $(\mathrm{B})$ serum triglycerides and non-esterified (free) fatty acids $\left({ }^{*} p<0.05\right)$. Data represents the mean \pm $\operatorname{SEM}(n=12)$. 
Citation: Bhathena J, Tomaro-Duchesneau C, Martoni C, Malhotra M, Kulamarva A, et al. (2012) Effect of Orally Administered Microencapsulated FA-Producing L. fermentum on Markers of Metabolic Syndrome: An In vivo Analysis. J Diabetes Metab S6:006. doi:10.4172/2155-6156.S6-006

Page 6 of 9

FA is a naturally found phenolic acid that has numerous beneficial properties. Recent studies suggest that FA has antitumor activity against breast cancer [30,31], liver cancer $[32,33]$ and is effective at preventing cancer induced by the exposure to carcinogenic compounds such as benzopyrene [34] and 4-nitroquinoline 1-oxide [35]. FA has also been demonstrated to have important antioxidant properties [1] while also reducing cell aging [2]. Keeping this in mind, we propose that FA can be used to modulate a number of biomarkers related to metabolic syndrome, specifically cardiovascular diseases, T2DM and obesity (Figure 7). Unfortunately, the oral delivery of free FA is hampered by its quick absorption in the jejunum, followed by its rapid excretion [36]. It has been demonstrated in a number of recent studies that some GIT bacterial strains produce FAE, an enzyme that can produce FA from natural substrates. The oral delivery of these bacteria, however, is impeded by the harsh conditions of the upper GIT, specifically the presence of bile and an acidic pH. Microencapsulation, specifically APA microencapsulation, has been successfully used to overcome the challenge of delivering bacterial cells through the GIT [11]. APA microencapsulation relies on a polyelectrolyte complexation mechanism for the association of alginate and PLL. Alginate is a natural biocompatible polymer, extracted from brown algae, that is increasingly being used in the biotechnology industry [14]. Alginate is an unbranched polysaccharide containing 1,4'-linked $\beta$-D-mannuronic acid and $\alpha$-L-guluronic acid blocks interdispersed with regions of the alternating structure, $\beta$-Lmannuronic acid- $\alpha$-L-guluronic acid blocks. PLL is a polypeptide made up of the amino acid L-lysine that is available in a variable number of chain lengths. The addition of this polymer leads to the formation of a capsule membrane that provides selective permeability and immunoprotection. The alginate bead could not withstand the harsh conditions of the GIT in the absence of PLL, which provides it with an increased mechanical stability. Previous research, by our group, investigated APA microencapsulation as a method to protect bacterial viability through a GIT transit, and demonstrated a significant 2.5 log difference in viability between free and APA microencapsulated L. fermentum following transit [11].

Previous research by our group has screened for FA production by probiotic bacteria [7,10]. For this research, L.f. 11976 was selected for its FAE activity. In vitro studies into the antioxidant properties of this strain were performed with relation to FA production. Following 48 hours of incubation, the antioxidant activity of L.f. 11976 was determined and correlated with a standard curve generated for FA. Using the curve generated for FA the activity correlated to $1931.83 \pm 10.07 \mu \mathrm{M}$ for the free cells, greater than that quantified by previous HPLC assays $[7,10]$. These results suggest that the antioxidant activity of L.f. 11976 may not be explained solely by its FA production, but by potential other molecules produced by this strain, which require further investigations. The demonstrated antioxidant activity as well as its previously demonstrated effects on hypercholesterolemia make it an ideal candidate for this study [7].

Feeding of a high lipid diet provides a dietary model of metabolic syndrome-cardiovascular diseases, T2DM and obesity-similar to that of a Western Diet. A number of risk factors are involved in metabolic syndrome, including insulin resistance, hyperglycemia, hyperinsulinemia, chronic systemic inflammation and hypertriglyceridemia, to name a few. An overload of fat to the liver leads to the disruption of metabolic and glucose uptake pathways, potentially leading to an enhanced rate of de novo lipogenesis and triglyceride synthesis, ultimately

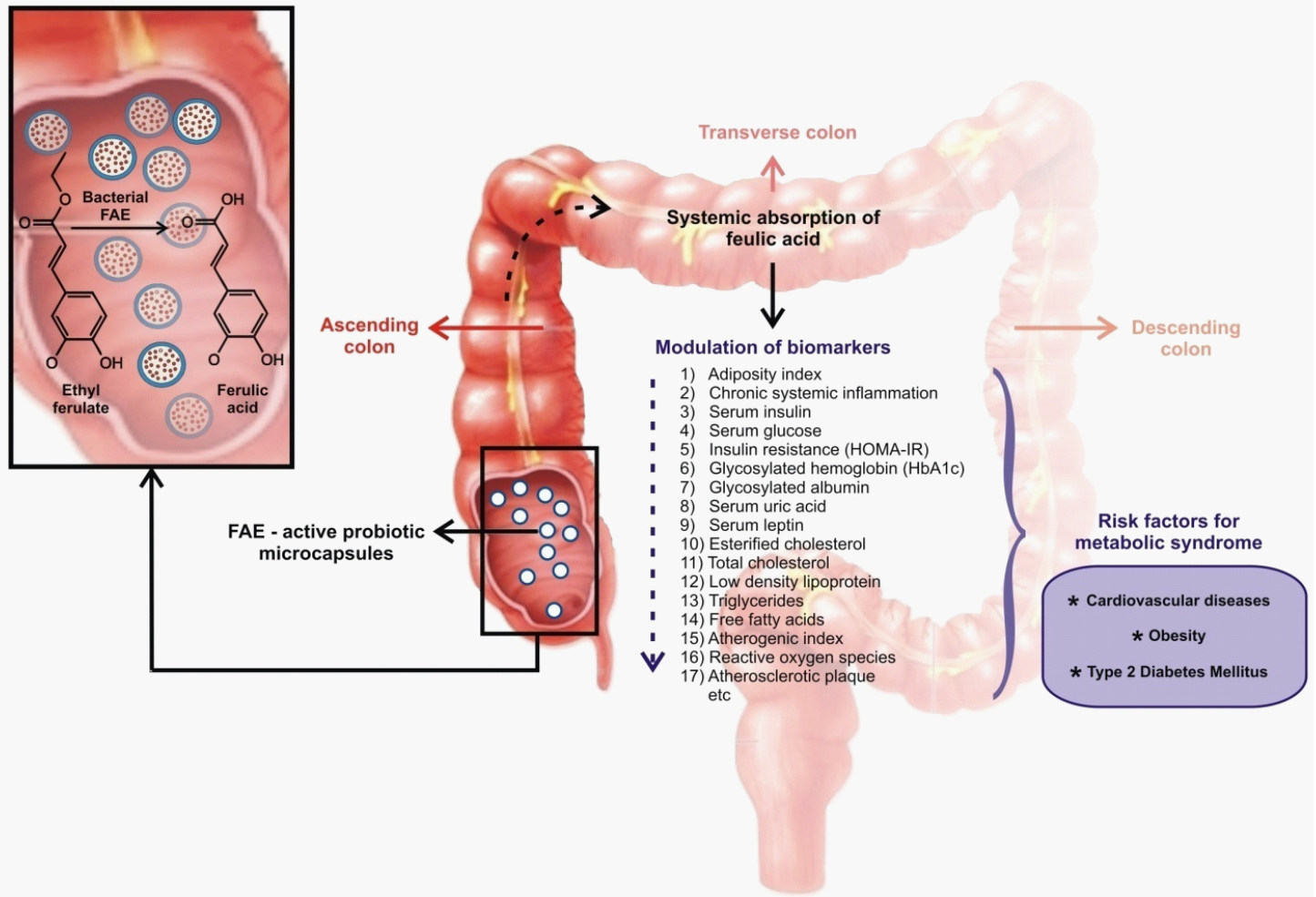

Figure 7: FA is proposed to modulate markers of metabolic syndrome, presenting a number of different mechanisms of action by which it can prove beneficial. Probiotic microcapsules, in the ascending colon, release FA due to FAE enzymatic activity, leading to the systemic absorption of FA. FA has been shown, in the presented research and in other works, to modulate a number of markers that play an important role in metabolic syndrome-cardiovascular diseases, obesity and T2DM. 
inducing insulin resistance. The presented study uses a hamster model fed a hyperlipidemic hypercholesterolemic diet, leading to the induction of moderate insulin resistance and elevation of serum glucose. Future research may also want to investigate to what extent markers of metabolic syndrome are restored following probiotic treatment, using a comparative group fed a standard diet. One could also investigate the potential effect(s) of treatment on animals consuming a standard diet.

Following 10 weeks of treatment with APA microencapsulated L.f. 11976 the serum glucose levels were only numerically disparate in the treated animals when compared to the control group. Furthermore, no significant difference in animal body mass was noted, suggesting that a longer treatment period may be necessary to significantly alter hyperglycemia and obesity. On the other hand, insulin levels were significantly lower in the treatment group than the control, suggesting a control of hyperinsulinemia by the probiotic formulation. In addition, insulin resistance, as determined using HOMA-IR, was alleviated significantly ( $p=0.0096)$, demonstrating the ability of microencapsulated L.f. 11976 to improve the sensitivity of the peripheral tissues to insulin.

The United Kingdom Prospective Diabetes Study demonstrated that a $1 \%$ reduction of $\mathrm{HbAlc}$ is associated with a $35 \%$ reduction in macrovascular endpoints, an $18 \%$ reduction in myocardial infarction, and a $17 \%$ reduction in all-cause mortality [37]. In our study, the HbAlc levels, following 10 weeks of treatment, demonstrated only a non-significant difference $(8.41 \pm 1.40 \%)$ for the treated and $(12.85 \pm 1.59 \%)$ for the control animals $(p=0.058)$. One must keep in mind, however, that total $\mathrm{HbAlc}$ reveals the percentage of circulating hemoglobin that has chemically reacted with glucose, reflecting the blood glucose levels over the 120 days preceding the test [38], and is, therefore, unaffected by short term fluctuations in blood glucose levels present in a short $(70$ days) study like this one. Furthermore, the non-significant decrease in $\mathrm{HbAlc}$ levels observed in our study may reflect the need for the longer administration or a higher dose of probiotic to demonstrate an adequate and long-term reduction of hyperglycemia.

Serum GA levels have been shown to be a better alternative marker of glycemic control in patients with T2DM [39] since it is free of interference from endogenous glycated amino acids and is unaffected by changes in albumin concentration. In the presented study, hamsters treated with microencapsulated L.f. 11976 demonstrated significantly lower GA concentrations than the control group $(p=0.00013)$. Furthermore, control animals had significantly increased TC, TG, and NEFA levels. Dyslipidemia is often one of the first risk factors identified and observed in pre-diabetic patients [40], suggesting a link between dyslipidemia and T2DM initiation. For example, it has been reported that GA contributes to dyslipidemia in diabetes (and renal insufficiency) [41] and GA modification impairs the Low-Density Lipoprotein (LDL) receptor-clearance mechanism [42]. Hence, even a small decrease in GA (such as that observed in this study) and TC, TG and NEFA levels may be sufficient to stop the triggering of pathogenic mechanisms underlying accelerated atherosclerosis, nephropathy and retinopathy [43]. Future studies should take into consideration that GA measurements reflect glycemic control for only two weeks preceding the assay, and so should be taken more frequently throughout the study.

Our results demonstrate a significant reduction in serum NEFA levels in the animal group treated with microencapsulated L.f. 11976, following 10 weeks of treatment $(p=0.029)$. Recent studies support the idea of a correlation between elevated NEFA and peripheral insulin resistance [44]. In obese individuals, serum NEFA levels are chronically elevated, contributing to insulin resistance at the skeletal muscle level. Furthermore, these may have additional actions in the liver and pan- creas, contributing to the development of T2DM [45]. Hence, NEFA consist of an important therapeutic target that our probiotic formulation successfully modulated.

The administration of microencapsulated L.f. 11976 also reduced the accumulated levels of advanced glycosylation end-products, produced by non-enzymatic glycosylation of proteins (Maillard reaction) [46], as indicated by the decrease in total HbA1c and albumin. Glycosylated end-products are present in high levels in T2DM patients, stimulating macrophages to secrete inflammatory cytokines and growth factors, impairing normal macrophage function, hence, increasing susceptibility to infection [47]. Similarly, inflammatory markers, particularly CRP, play an important role in cardiovascular disease as they are linked with an increased risk of atherosclerosis, myocardial infarction and diabetes $[48,49]$. These findings suggest an association between glycemic control and systemic inflammation in people with established diabetes, specifically with HbA1c levels [48]. In the presented study, administration of microencapsulated L.f. 11976 decreased slightly, although not significantly, serum CRP levels $(p=0.61)$, which may be explained by the high levels of HbAlc observed.

Hyperuricemia also demonstrates a strong correlation with insulin resistance, and cardiovascular disease, but not with blood glucose levels [50]. The link between insulin resistance and hyperuricemia, elucidated by Facchini et al. demonstrates that urinary uric acid clearance decreases in proportion to an increase in insulin resistance, manifesting as an increase in serum uric acid concentrations [51]. Our results demonstrate simultaneous reduction of serum uric acid and insulin resistance in hamsters receiving microencapsulated L.f. 11976 when compared to control animals. Previous research has also noted that elevated serum leptin levels, a neuroregulatory peptide that regulates lipid, glucose and insulin, contribute to increased serum uric acid levels [52]. A majority of patients with T2DM have elevated leptin levels, associated with that seen in obese subjects [53]. In our study, there was no significant difference in body weight $(p=0.178)$ between the treated and control animals, even though the serum leptin levels were significantly lower in the treated animals $(p=0.0476)$. Previous research has demonstrated a strong positive correlation between serum leptin concentration and the percentage of body fat, as we also demonstrate with a decreased adiposity index [53]. As aforementioned, despite the difference in serum leptin levels, no significant difference in food consumption or body mass was noted between the two groups. Previous research has demonstrated that obese subjects may have decreased sensitivity to leptin and require larger doses of leptin to induce weight loss, explaining the phenomena seen in the presented work [53].

The consumption of functional foods containing probiotics has been proposed as one of the best approaches to treat and/or inhibit the prevalence of T2DM, obesity and cardiovascular diseases. It has previously been demonstrated that probiotic lactic acid bacteria can control glucose levels and significantly delay the onset of hyperglycemia, hyperinsulinemia, dyslipidemia, glucose intolerance and oxidative stress [54,55]. The supplementation of probiotic L. acidophilus and $L$. casei with dahi cultures, increased the efficacy of dahi in suppressing streptozotocin-induced diabetes in rats by inhibiting insulin depletion, diabetic dyslipidemia, lipid peroxidation and nitrite formation [54]. FA-producing microencapsulated lactic acid bacteria have been shown to liberate FA in the gut $[8,11]$. Currently, it is not clear exactly how FA is impacting metabolic syndrome, as presented in this study. However as shown in Figure 7, the mechanism(s) of action by which FA exerts its beneficial effects has been hypothesized, with a number of biomark- 
Citation: Bhathena J, Tomaro-Duchesneau C, Martoni C, Malhotra M, Kulamarva A, et al. (2012) Effect of Orally Administered Microencapsulated FA-Producing L. fermentum on Markers of Metabolic Syndrome: An In vivo Analysis. J Diabetes Metab S6:006. doi:10.4172/2155-6156.S6-006

ers involved that play important roles in metabolic syndrome. Future research should focus on the potential mechanisms of action and further explore the role of FA in all of the markers presented. The exact role of FA in modulating the markers described in this research will be investigated further using both in vitro and in vivo studies, focusing on both the metabolic and anti-inflammatory effects of this molecule. The objective of this research was to demonstrate the use of a FA-producing probiotic on the modulation of metabolic markers, with mechanistic studies to follow. Future animal work should also be undertaken using other models of metabolic syndrome and T2DM.

\section{Conclusion}

The presented study investigated the effects of feeding microencapsulated live probiotic L.f. 11976 on biomarkers of metabolic syndrome, entailing cardiovascular diseases, obesity and T2DM. The evidence for a possible role of dietary FA for the treatment and prevention of these disorders is presented and discussed. Keeping in view the gaps in these studies, the various mechanisms by which FA may improve the control of glucose tolerance, lipid metabolism, body mass and other markers presented here, need to be further investigated.

\section{Acknowledgments/Disclosures}

The authors acknowledge support by grants from the Natural Science and Engineering Research Council (NSERC): Idea to Innovation (I2I) Grant sponsored by Micropharma Ltd., Montreal, Canada (Grant \# 205811) (to SP).JB is supported by a Doctoral Research Awardfrom the Canadian Institutes of Health Research (CIHR). The authors would also like to acknowledge a Canada Graduate Scholarship from NSERC (to CM), Alexander Graham Bell Canada Graduate Scholarship from NSERC (to AK, AP and CTD), a Doctoral FRSQ Scholarship (to MM) and a Post Graduate Scholarship from NSERC (to AMU)

SP and JB have a conflict of interest with Micropharma Ltd., Montreal, Cana$\mathrm{da}$; the technology mentioned in this research is optioned to Micropharma Ltd., for commercialization. None of the other authors have any personal or financial conflict of interest. The design and conduct of the study; collection, management, analysis, and interpretation of the data; and preparation of the manuscript was independent of the funding organizations or sponsors. The manuscript was reviewed and approved by the sponsor.

\section{References}

1. Rice-Evans CA, Miller NJ, Paganga G (1996) Structure-antioxidant activity relationships of flavonoids and phenolic acids. Free Radic Biol Med 20: 933956.

2. Lombard DB, Chua KF, Mostoslavsky R, Franco S, Gostissa M, et al. (2005) DNA repair, genome stability, and aging. Cell 120: 497-512.

3. Hu CT, Wu JR, Cheng CC, Wang S, Wang HT, et al. (2011) Reactive oxygen species-mediated PKC and integrin signaling promotes tumor progression of human hepatoma HepG2. Clin Exp Metastasis 28: 851-863.

4. Ishii N, Fujii M, Hartman PS, Tsuda M, Yasuda K, et al. (1998) A mutation in succinate dehydrogenase cytochrome b causes oxidative stress and ageing in nematodes. Nature 394: 694-697.

5. Sonta T, Inoguchi T, Tsubouchi H, Sekiguchi N, Kobayashi K, et al. (2004) Evidence for contribution of vascular $\mathrm{NAD}(\mathrm{P}) \mathrm{H}$ oxidase to increased oxidative stress in animal models of diabetes and obesity. Free Radical Biol Med 37: $115-123$

6. Donath MY, Shoelson SE (2011) Type 2 diabetes as an inflammatory disease. Nat Rev Immunol 11: 98-107.

7. Bhathena J, Kulamarva A, Urbanska AM, Martoni C, Prakash S (2007) Microencapsulated bacterial cells can be used to produce the enzyme feruloyl esterase: preparation and in-vitro analysis. Appl Microbiol Biotechnol 75: 10231029.

8. Bhathena J, Kulamarva A, Martoni C, Urbanska AM, Prakash S (2008) Preparation and in vitro analysis of microencapsulated live Lactobacillus fermentum 11976 for augmentation of feruloyl esterase in the gastrointestinal tract. Biotechnol Appl Biochem 50: 1-9.

9. Bhathena J, Martoni C, Kulamarva A, Urbanska AM, Malhotra M, et al. (2009)
Orally delivered microencapsulated live probiotic formulation lowers serum lipids in hypercholesterolemic hamsters. J Med Food 12: 310-319.

10. Tomaro-Duchesneau C, Saha S, Malhotra M, Coussa-Charley M, Al-Salam $\mathrm{H}$, et al. (2012) Lactobacillus fermentum NCIMB 5221 has a greater ferulic acid production compared to other ferulic acid esterase producing Lactobacilli. International Journal of Probiotics and Prebiotics 7.

11. Tomaro-Duchesneau C, Saha S, Malhotra M, Coussa-Charley M, Kahouli I, et al. (2012) Probiotic Ferulic Acid Esterase Active Lactobacillus fermentum NCIMB 5221 APA Microcapsules for Oral Delivery: Preparation and in Vitro Characterization. Pharmaceuticals 5: 236-248.

12. Lai KK, Lorcan GL, Gonzalez CF (2009) Biochemical properties of two cinnamoyl esterases purified from a Lactobacillus johnsonii strain isolated from stool samples of diabetes-resistant rats. Appl Environ Microbiol 75: 5018-5024.

13. Metchnikoff É (1907) The Prolongation of Life: Optimistic Studies,New York \& London : G.P. Putnam's Sons.

14. Prakash S, Tomaro-Duchesneau C, Saha S, Cantor A (2011) The gut microbiota and human health with an emphasis on the use of microencapsulated bacterial cells. J Biomed Biotechnol 2011: 981214.

15. Narayan KM, Boyle JP, Geiss LS, Saaddine JB, Thompson TJ (2006) Impact of recent increase in incidence on future diabetes burden: U.S., 2005-2050. Diabetes Care 29: 2114-2116.

16. Lavie CJ, Milani RV (2003) Obesity and cardiovascular disease: the hippocrates paradox? J Am Coll Cardiol 42: 677-679.

17. Adisakwattana S, Moonsan P, Yibchok-anun S (2008) Insulin-releasing properties of a series of cinnamic acid derivatives in vitro and in vivo. J Agric Food Chem 56: 7838-7844.

18. Taylor BA, Phillips SJ (1996) Detection of obesity QTLs on mouse chromosomes 1 and 7 by selective DNA pooling. Genomics 34: 389-398.

19. de Roos B, Rucklidge G, Reid M, Ross K, Duncan G, et al. (2005) Divergent mechanisms of cis9, trans11-and trans10, cis12-conjugated linoleic acid affecting insulin resistance and inflammation in apolipoprotein $E$ knockout mice: a proteomics approach. FASEB J 19: 1746-1748.

20. FAO and WHO (2001) Health and nutritional properties of probiotics in food including powder milk with live lactic acid bacteria. Argentina.

21. Branton WB, Jones ML, Tomaro-Duchesneau C, Martoni CJ, Prakash S (2011) In vitro characterization and safety of the probiotic strain Lactobacillus reuter cardioviva NCIMB 30242. International Journal of Probiotics and Prebiotics 6 : $1-12$

22. Matés JM, Pérez-Gómez C, Núñez de Castro I, Asenjo M, Márquez J (2002) Glutamine and its relationship with intracellular redox status, oxidative stress and cell proliferation/death. Int J Biochem Cell Biol 34: 439-458.

23. Martoni C, Bhathena J, Urbanska AM, Prakash S (2008) Microencapsulated bile salt hydrolase producing Lactobacillus reuteri for oral targeted delivery in the gastrointestinal tract. Appl Microbiol Biotechnol 81: 225-233.

24. Tanaka H, Doesburg K, Iwasaki T, Mierau I (1999) Screening of Lactic Acid Bacteria for Bile Salt Hydrolase Activity. J Dairy Sci 82: 2530-2535.

25. Pfeiler EA, Klaenhammer TR (2009) Role of Transporter proteins in bile tolerance of Lactobacillus acidophilus. Appl Environ Microbiol 75: 6013-6016.

26. Srinivasan M, Sudheer AR, Menon VP (2007) Ferulic Acid: therapeutic potential through its antioxidant property. J Clin Biochem Nutr 40: 92-100.

27. Balasubashini MS, Rukkumani R, Menon VP (2003) Protective effects of ferulic acid on hyperlipidemic diabetic rats. Acta Diabetol 40: 118-122.

28. Balasubashini MS, Rukkumani R, Viswanathan P, Menon VP (2004) Ferulic acid alleviates lipid peroxidation in diabetic rats. Phytother Res 18: 310-314.

29. Atsuyo F, Hideyuki S, Asako D, Kunihisa O, Shohei M, et al. (2008) Ferulic acid prevents pathological and functional abnormalities of the kidney in Otsuka Long-Evans Tokushima Fatty diabetic rats. Diabetes Res Clin Pract 79: 11-17.

30. Chang CJ, Chiu JH, Tseng LM, Chang CH, Chien TM, et al. (2006) Modulation of HER2 expression by ferulic acid on human breast cancer MCF7 cells. Eur $J$ Clin Invest 36: 588-596.

31. Kampa M, Alexaki VI, Notas G, Nifli AP, Nistikaki A, et al. (2004) Antiproliferative and apoptotic effects of selective phenolic acids on T47D human breast cancer cells: potential mechanisms of action. Breast Cancer Res 6: R63-R74. 
Citation: Bhathena J, Tomaro-Duchesneau C, Martoni C, Malhotra M, Kulamarva A, et al. (2012) Effect of Orally Administered Microencapsulated FA-Producing L. fermentum on Markers of Metabolic Syndrome: An In vivo Analysis. J Diabetes Metab S6:006. doi:10.4172/2155-6156.S6-006

32. Lee YS (2005) Role of NADPH oxidase-mediated generation of reactive oxygen species in the mechanism of apoptosis induced by phenolic acids in HepG2 human hepatoma cells. Arch Pharm Res 28:1183-1189.

33. Taniguchi H, Hosoda A, Tsuno T, Maruta Y, Nomura E (1999) Preparation of ferulic acid and its application for the synthesis of cancer chemopreventive agents. Anticancer Res 19: 3757-3761.

34. Lesca P (1983) Protective effects of ellagic acid and other plant phenols on benzo[a]pyrene-induced neoplasia in mice. Carcinogenesis 4: 1651-1653.

35. Tanaka T, Kojima T, Kawamori T, Wang A, Suzui M, et al. (1993) Inhibition of 4-nitroquinoline-1-oxide-induced rat tongue carcinogenesis by the naturally occurring plant phenolics caffeic, ellagic, chlorogenic and ferulic acids. Carcinogenesis 14: 1321-1325.

36. Zhao Z, Egashira Y, Sanada H (2003) Digestion and absorption of ferulic acid sugar esters in rat gastrointestinal tract. J Agric Food Chem 51: 5534-5539.

37. UK Prospective Diabetes Study Group (1998) Tight blood pressure contro and risk of macrovascular and microvascular complications in type 2 diabetes: UKPDS 38. BMJ 317: 703-713.

38. Gabbay KH, Hasty K, Breslow JL, Ellison RC, Bunn HF, et al. (1977) Glycosylated hemoglobins and long-term blood glucose control in diabetes mellitus. J Clin Endocrinol Metab 44: 859-864.

39. Inaba M, Okuno S, Kumeda Y, Yamada S, Imanishi Y, et al. (2007) Glycated albumin is a better glycemic indicator than glycated hemoglobin values in hemodialysis patients with diabetes: effect of anemia and erythropoietin injection. J Am Soc Nephrol 18: 896-903.

40. Dagogo-Jack S (2005) Primary prevention of cardiovascular disease in prediabetes: the glass is half full and half empty. Diabetes Care 28: 971-972.

41. Bucala R, Makita Z, Vega G, Grundy S, Koschinsky T, et al. (1994) Modification of low density lipoprotein by advanced glycation end products contributed to the dyslipidemia of diabetes and renal insufficiency. Proc Natl Acad Sci U S A 91: $9441-9445$.

42. Aronson D, Rayfield EJ (2002) How hyperglycemia promotes atherosclerosis: molecular mechanisms. Cardiovas Diabetol 1: 1.

43. Schalkwijk CG, Miyata T (2012) Early- and advanced non-enzymatic glycation in diabetic vascular complications: the search for therapeutics. Amino Acids 42: $1193-1204$

44. Barazzoni R, Zanetti M, Cappellari G, Semolic A, Boschelle M, et al. (2012) Fatty acids acutely enhance insulin-induced oxidative stress and cause insulin resistance by increasing mitochondrial reactive oxygen species (ROS) generation and nuclear factor- $\mathrm{KB}$ inhibitor (IKB)-nuclear factor-kB (NFKB) activation in rat muscle, in the absence of mitochondrial dysfunction. Diabetologia 55: 773-782

45. Tushuizen ME, Bunck MC, Pouwels PJ, Bontemps S, van Waesberghe JH, et al. (2007) Pancreatic fat content and beta-cell function in men with and without type 2 diabetes. Diabetes Care 30: 2916-2921.

46. Maillard LC (1912) Action des acides amines sur les sucres; formation des melanoides par voie methodique. Comptes Rendus Des Seances De L'Academie des Sciences 154: 66-68.

47. Liu BF, Miyata S, Kojima H, Uriuhara A, Kusunoki H, et al. (1999) Low phagocytic activity of resident peritoneal macrophages in diabetic mice: relevance to the formation of advanced glycation end products. Diabetes 48: 2074-2082.

48. King DE, Mainous AG, Buchanan TA, Pearson WS (2003) C-reactive protein and glycemic control in adults with diabetes. Diabetes Care 26: 1535-1539.

49. Ridker PM, Glynn RJ, Hennekens CH (1998) C-Reactive Protein Adds to the predictive value of total and $\mathrm{HDL}$ cholesterol in determining risk of first myocardial infarction. Circulation 97: 2007-2011.

50. Lin JD, Chiou WK, Chang HY, Liu FH, Weng HF (2007) Serum uric acid and leptin levels in metabolic syndrome: a quandary over the role of uric acid. Metabolism 56: 751-756.

51. Facchini F, Chen YD, Hollenbeck CB, Reaven GM (1991) Relationship between resistance to insulin-mediated glucose uptake, urinary uric acid clearance, and plasma uric acid concentration. JAMA 266: 3008-3011.

52. Matsubara M, Chiba H, Maruoka S, Katayose S (2002) Elevated Serum leptin concentrations in women with hyperuricemia. J Atheroscler Thromb 9: 28-34.

53. Considine RV, Sinha MK, Heiman ML, Kriauciunas A, Stephens TW, et al (1996) Serum immunoreactive-leptin concentrations in normal-weight and obese humans. N Engl J Med 334: 292-295

54. Yadav H, Jain S, Sinha PR (2008) The effect of probiotic dahi containing Lactobacillus acidophilus and Lactobacillus casei on gastropathic consequences in diabetic rats. J Medic Food 11:62-68.

55. Yadav H, Jain S, Sinha PR (2007) Antidiabetic effect of probiotic dahi containing Lactobacillus acidophilus and Lactobacillus casei in high fructose fed rats. Nutrition 23: 62-68.

This article was originally published in a special issue, Metabolomics: Diabetics handled by Editor(s). Dr. Ippei Kanazawa, Shimane University Faculty of Medicine, Japan 